\title{
Avaliação de pacientes críticos com suspeita de sepse em um hospital universitário
}

\author{
Evaluation of critical patients with suspected sepsis in a university hospital \\ Evaluación de pacientes críticos con sospecha de sepsis en un hospital universitario
}

Phydel Palmeira Carvalho

ORCID: https://orcid.org/0000-0001-6229-943X

Universidade Estadual da Bahia, Brasil

E-mail: phydel_ppc@yahoo.com.br

Murilo de Jesus Porto

ORCID: https://orcid.org/0000-0003-2339-8173

Universidade do Estado da Bahia, Brasil

E-mail: murilo.porto@ hotmail.com

Jamile Fontenelle Barros

ORCID: https://orcid.org/0000-0002-7438-8525 Universidade Federal de Sergipe, Brasil

E-mail: jamile.jfb@outlook.com

Alessana Silva Magalhães

ORCID: https://orcid.org/0000-0002-7854-3772 Universidade Federal de Sergipe, Brasil

E-mail: alessana_magalhaes@hotmail.com

Luann Soares Nunes

ORCID: https://orcid.org/0000-0001-5190-5318 Universidade Federal de Sergipe, Brasil

E-mail: luann.soares@hotmail.com

Icaro Cruz Silva

ORCID: https://orcid.org/0000-0002-4581-8987 Universidade Federal de Sergipe, Brasil

E-mail: icarocrz@gmail.com

Isis Layane Silva de Abreu

ORCID: https://orcid.org/0000-0002-8595-3243 Universidade Federal de Sergipe, Brasil

E-mail: isis.ls.abreu@hotmail.com

Gilvania Nascimento Souza

ORCID: https://orcid.org/0000-0002-4715-4088 Centro Universitário UNIAGES, Brasil E-mail: souza.gilvania@yahoo.com.br

José Melquiades de Rezende Neto

ORCID: https://orcid.org/0000-0002-4955-8529 Universidade Federal de Sergipe, Brasil E-mail: jmkide@gmail.com

Thiago da Silva Mendes

ORCID: https://orcid.org/0000-0002-9221-9274 Universidade Federal de Sergipe, Brasil E-mail: thiago.mendes@ebserh.gov.br

\begin{abstract}
Resumo
Sepse é uma das principais causas de morte em pacientes graves. A antibioticoterapia rápida e adequada é de grande importância para esses pacientes, mas longos tratamentos de antimicrobianos são indesejáveis por aumentarem a resistência aos antibióticos. Esta pesquisa tem como objetivo geral avaliar os dados clínicos e epidemiológicos de pacientes críticos com suspeita de sepse em um Hospital Universitário no interior de Sergipe. Foi realizado um estudo epidemiológico longitudinal exploratório prospectivo, quantitativo e descritivo nas unidades críticas do Hospital Universitário de Lagarto (Sergipe - Brasil). A pesquisa foi aprovada pelo conselho de revisão institucional — Comitê de Ética CAAE no 16847919.8.0000.5546 - Número do parecer: 3.496.684 — da Fundação Universidade Federal de Sergipe (UFS). Foram acompanhados 48 pacientes através de busca ativa diária de novos casos suspeitos de sepse. Quanto aos resultados, 52,1\% (25) dos pacientes eram mulheres, sendo a média de idade de 64,29 anos. A média do tempo de permanência hospitalar foi de 32,02 dias. Mais da metade dos casos suspeitos de sepse, 52,1\% (25) foi secundária a infecções pulmonares e trato respiratório e 22,9\% (11) relacionados à foco urinário. Os tratamentos antimicrobianos mais prescritos foram: 21,3\% Meropenem; 13,9\% Piperacilina + Tazobactam; 13,1\% Ceftriaxona. Do total de pacientes avaliados, 45,8\% (22) foram a óbito. Conclui-se que pacientes com períodos prolongados de internação são mais vulneráveis à sepse. Com isso, a taxa de mortalidade identificada é preocupante e reforça a
\end{abstract}


necessidade de identificar precocemente os fatores associados ao desenvolvimento da sepse e, por isso, a importância de implantar um protocolo de sepse nas instituições.

Palavras-chave: Sepse; Antibioticoterapia; Paciente crítico.

Abstract

Sepsis is a major cause of death in critically ill patients. Rapid and adequate antibiotic therapy is of great importance for these patients, but long-term antimicrobial treatments are undesirable because they increase resistance to antibiotics. This research aims to evaluate the clinical and epidemiological data of critically ill patients with suspected sepsis in a University Hospital in the interior of Sergipe. A prospective, quantitative and descriptive longitudinal exploratory epidemiological study was carried out in the critical units of the Hospital Universitário de Lagarto (Sergipe - Brazil). The research was approved by the institutional review board - CAAE Ethics Committee No. 16847919.8.0000.5546 - Opinion number: 3,496,684 - from the Federal University of Sergipe Foundation (UFS). 48 patients were followed through an active daily search for new suspected cases of sepsis. As for the results, 52.1\% (25) of the patients were women, with a mean age of 64.29 years. The average length of hospital stay was 32.02 days. More than half of the suspected cases of sepsis, $52.1 \%$ (25) were secondary to pulmonary and respiratory tract infections and $22.9 \%$ (11) related to the urinary focus. The most prescribed antimicrobial treatments were: $21.3 \%$ Meropenem; 13.9\% Piperacillin + Tazobactam; 13.1\% Ceftriaxone. Of the total number of patients evaluated, 45.8\% (22) died. It is concluded that patients with prolonged periods of hospitalization are more vulnerable to sepsis. As a result, the mortality rate identified is worrying and reinforces the need to early identify the factors associated with the development of sepsis and, therefore, the importance of implementing a sepsis protocol in institutions.

Keywords: Sepsis; Antibiotic therapy; Critical patient.

\begin{abstract}
Resume
La sepsis es una de las principales causas de muerte en pacientes críticamente enfermos. La terapia antibiótica rápida y adecuada es de gran importancia para estos pacientes, pero los tratamientos antimicrobianos a largo plazo son indeseables porque aumentan la resistencia a los antibióticos. Esta investigación tiene como objetivo evaluar los datos clínicos y epidemiológicos de pacientes críticos con sospecha de sepsis en un Hospital Universitario del interior de Sergipe. Se realizó un estudio epidemiológico exploratorio longitudinal prospectivo, cuantitativo y descriptivo en las unidades críticas del Hospital Universitário de Lagarto (Sergipe - Brasil). La investigación fue aprobada por la junta de revisión institucional - Comité de Ética CAAE No. 16847919.8.0000.5546 - Número de opinión: 3.496 .684 - de la Fundación Universidad Federal de Sergipe (UFS). Se siguió a 48 pacientes mediante una búsqueda activa diaria de nuevos casos sospechosos de sepsis. En cuanto a los resultados, el 52,1\% (25) de los pacientes eran mujeres, con una edad media de 64,29 años. La duración media de la estancia hospitalaria fue de 32,02 días. Más de la mitad de los casos sospechosos de sepsis, el 52,1\% (25) fueron secundarios a infecciones pulmonares y del tracto respiratorio y el 22,9\% (11) relacionados con el foco urinario. Los tratamientos antimicrobianos más prescritos fueron: $21,3 \%$ de meropenem; $13,9 \%$ de piperacilina + tazobactam; $13,1 \%$ de ceftriaxona. Del total de pacientes evaluados falleció el $45,8 \%$ (22). Se concluye que los pacientes con periodos prolongados de hospitalización son más vulnerables a la sepsis. En consecuencia, la tasa de mortalidad identificada es preocupante y refuerza la necesidad de identificar precozmente los factores asociados al desarrollo de la sepsis y, por tanto, la importancia de implementar un protocolo de sepsis en las instituciones.
\end{abstract}

Palabras clave: Sepsis; Terapia con antibióticos; Paciente crítico.

\title{
1 Introdução
}

Sepse é definida pelo Instituto Latino Americano de Sepse - ILAS (2018), como "a presença de disfunção ameaçadora à vida em decorrência da presença de resposta desregulada à infecção". As principais disfunções orgânicas são: Hipotensão (PAS < $90 \mathrm{mmHg}$ ou PAM $<65 \mathrm{mmHg}$ ou queda de PA > $40 \mathrm{mmHg})$; Oligúria $(\leq 0,5 \mathrm{~mL} / \mathrm{Kg} / \mathrm{h})$ ou elevação da creatinina (>2mg/dL); Relação $\mathrm{PaO}_{2} / \mathrm{FiO}_{2}<300$ ou necessidade de $\mathrm{O}_{2}$ para manter $\mathrm{SpO}_{2}>90 \%$; Contagem de plaquetas < 100.000/mm ou redução de 50\% no número de plaquetas. Em 2016 foi elaborado o Terceiro Consenso de Definição de Sepse e Choque Séptico (sepsis-3) pela European Society of Intensive Care Medicine (ESICM) e pela Society of Critical Care Medicine (SCCM), documento que redefine o que é sepse. O diagnóstico de sepse não está mais focado na SIRS e passa a ganhar força um novo Score, conhecido como Sequential Organ Failure Assessment - SOFA (Singer et al., 2016).

Uma outra alteração no documento sepsis-3, foi a não utilização do termo "sepse grave", pois todos os casos de sepse passam a ser considerados graves e a nova definição de "choque séptico" como "um subgrupo dos pacientes com sepse que apresentam acentuada anormalidade circulatórias, celulares e metabólicas e associadas com maior risco de morte do que a sepse isoladamente". 
Os critérios diagnósticos de choque séptico são: a "necessidade de vasopressor para manter uma pressão arterial média acima de $65 \mathrm{mmHg}$ após a infusão adequada de fluidos, associada a nível sérico de lactato acima de 2mmol/L” (ILAS, 2018).

No Brasil, existe uma análise variável para qual definição de sepse utilizar. Depende dos recursos disponíveis no serviço público para classificar e tratar, por exemplo, a dosagem de lactato, a disponibilidade de antibióticos de amplo espectro e vasopressores e profissionais de saúde com pouca ou nenhuma formação formal no manejo de pacientes com sepse (Machado et al, 2016). A sepse é um problema global de saúde pública e deve ser identificado o mais precoce, investindo em educação continuada, investimento em detecção, infraestrutura e início rápido do tratamento.

Assim, na presença de uma disfunção orgânica sem outra explicação plausível e com foco infeccioso presumível, o diagnóstico de sepse deve ser feito e o pacote de tratamento iniciado imediatamente após a identificação (Cruz \& Macedo, 2016).

A terapia para sepse bacteriana, realizada de maneira empírica devido à incerteza diagnóstica em ambiente hospitalar, leva ao uso abusivo de antibióticos, ao aumento dos custos do tratamento e, principalmente, ao risco de resistência bacteriana. Uma das maneiras mais eficazes de se reduzir o desenvolvimento de resistência bacteriana é a suspensão precoce dos antimicrobianos nos casos de infecções não confirmadas ou pouco prováveis. Além disso, é importante estudar a redução do tempo e dos custos de internações hospitalares, garantindo a segurança do paciente.

Considerando que a duração reduzida do tratamento com antibióticos pode conter o surgimento de bactérias multirresistentes em unidades de terapia intensiva e clínicas hospitalares, uma abordagem mais racional do problema é concentrar-se no uso adequado de antimicrobianos potentes, particularmente na UTI, onde grande parte desse uso incorreto ocorre (Barros et al., 2016).

A hemocultura é o padrão-ouro para diagnóstico microbiológico para confirmar a infecção e indicar de forma mais racional os antimicrobianos apropriados para o tratamento (Singer et al., 2016). O escore SOFA é considerado padrão-ouro no diagnóstico da sepse e está relacionado a maior mortalidade; todavia não é prático, pois envolve parâmetros laboratoriais que nem sempre são coletados imediato a suspeita (plaquetas, creatinina, bilirrubinas, $\mathrm{PaO} 2$ ). A antibioticoterapia rápida e adequada é de grande importância em pacientes criticamente doentes, mas longo tratamento de antimicrobiano é indesejável por aumentar a resistência aos antibióticos (Azevedo \& Machado, 2019, Nascimento-Borges et al., 2020).

A partir dessas informações, o presente trabalho buscou avaliar pacientes críticos com suspeita de sepse em um Hospital Universitário do interior de Sergipe, verificando dados como idade, sexo, principais comorbidades, foco da infecção, tipo de diagnóstico, motivo e tempo de internação hospitalar, acompanhamento do tratamento farmacológico, como sedação, drogas vasoativas e antibioticoterapia, acompanhamento de exames laboratoriais, aplicação de escores de risco e taxa de mortalidade.

\section{Materiais e Métodos}

\section{Delineamento, duração, local do estudo e amostra}

Esta pesquisa, trata-se de um estudo exploratório descritivo com característica quantitativa, apresentando cunho epidemiológico longitudinal prospectivo, sendo realizado nas unidades críticas assistenciais em atendimentos em saúde, denominadas de Clínicas Amarela e Vermelha e UTI do Hospital Universitário de Lagarto (Sergipe - Brasil). Estas unidades prestam serviços públicos pelo Sistema Único de Saúde - SUS, com atendimento portas abertas de diversas especialidades, conta com o total de 78 leitos de internação cadastrados pelo CNES (Cadastro Nacional de Estabelecimentos de Saúde). O espaço do estudo é composto por: 11 leitos de clínica Amarela adulto; 3 leitos de clínica Vermelha adulto; e, 10 leitos de UTI (Totalizando 24 Leitos). A pesquisa foi realizada entre setembro de 2019 e fevereiro de 2020, acompanhando 48 pacientes com 
suspeita de sepse, através de busca ativa diária de novos casos suspeitos de sepse e acompanhando em dias alternados subsequentes de acordo metodologia.

\section{Protocolo do Estudo}

O protocolo do estudo foi aprovado pelo conselho de revisão institucional. O comitê de ética CAAE $n^{\circ}$ 16847919.8.0000.5546 - Número do parecer: 3.496.684 pela Fundação Universidade Federal de Sergipe (UFS), e autorização de coleta através do Termo de Consentimento Livre e Esclarecido - TCLE informado por escrito o que será obtido do paciente ou seus representantes legais antes da randomização, sendo necessário a impressão de 3 vias do Termo: uma para o pesquisador, outra para participante do estudo e outra em anexo ao prontuário.

Foram adotados como critérios de inclusão os pacientes com diagnóstico de sepse no dia da coleta para acompanhamento prospectivo, internados em área críticas, maiores de 18 anos. Os critérios de exclusão foram: 1) não suspeita de sepse; 2) pacientes menores de 18 anos; 3) gravidez conhecida; 4) infecção grave causada por vírus, parasitas, fungos ou micobactérias; e, 5) culturas negativas (sangue, urina, aspirado traqueal, ou lavado bronco alveolar) em pacientes com suspeita de sepse.

\section{Instrumentos para avaliação}

Os dados foram registrados em uma ficha de coleta incluindo informações sobre idade, sexo, data de admissão, motivo da admissão, data de início da bacteremia, foco da infecção, Escala de Coma de Glasgow, Escore SOFA (Sequential Organ Failure Assessment) e Escore Fisiológico Agudo Simplificado (SAPS3), comorbidades, acompanhamento da antibioticoterapia, sedação e uso de drogas vasoativas, data de alta hospitalar ou óbito.

Foram avaliados todos os pacientes críticos internados com suspeita de sepse durante sua permanência nas Clínicas Amarela, Vermelha e UTI em uso de Antibioticoterapia. Os dados foram coletados pelos pesquisadores (Farmacêutico residente e pelo grupo de pesquisa composto por acadêmicos do curso de medicina (Universidade Federal de Sergipe) e farmácia (Centro Universitário - UNIAGES).

Foi realizado um acompanhamento prospectivo, com coleta de dados pelo programa Aplicativo de Gestão para Hospitais Universitários - AGHU dos pacientes com suspeita de sepse. Na admissão dos pacientes (D0), foram utilizados como índices prognósticos o score SAPS3 e durante o acompanhamento dos pacientes em dias alternados (D0, D3, D5, D7, D10 e D14) seguindo a metodologia do trabalho para acompanhamento do paciente de Bouadma et al. (2010). Além disso, foi realizado o cálculo do escore SOFA para acompanhamento das disfunções orgânicas e o registro do uso de antibióticos de acordo o tempo de internação.

\section{Análise Estatística}

Todos os dados obtidos foram registrados no instrumento de coleta de dados. Os dados foram digitados em planilha eletrônica (Microsoft Excel®), conferidos e exportados para posterior análise estatística Statistical Package for the Social Science (SPSS), versão 20.0 (IBM®). A estatística descritiva foi apresentada pela média, desvio padrão e valores mínimos e máximos quando a variável apresentou distribuição normal. As variáveis categóricas foram expressas em porcentagem.

A estimativa da diferença de variáveis contínuas de distribuição normal foi realizada por teste não-paramétrico Quiquadrado, porém não houve diferença significativa. Para a escalas de gravidade foi utilizado teste de comparação de médias Scott Knott. 


\section{Resultados}

Foram avaliadas 48 internações, onde 52,1\% (25) eram mulheres e 47,9\% (23) eram homens. A média de idade foi 64,29 anos $( \pm 16,159)$. A média de tempo de permanência hospitalar foi de 32,02 dias.

Tabela 1. Distribuição do sexo e idade dos sujeitos da pesquisa $(n=48)$.

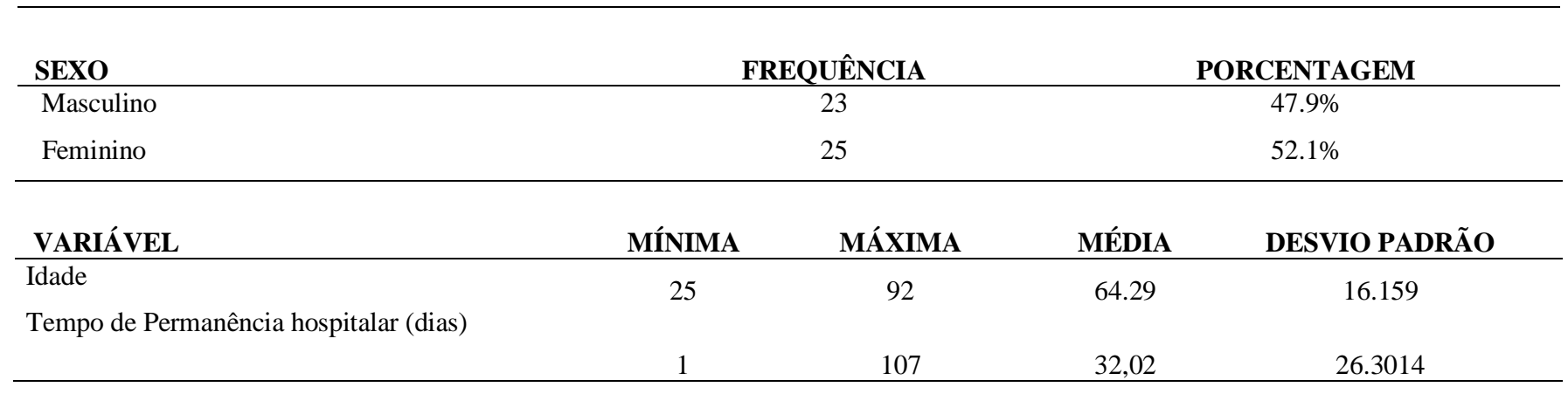

Fonte: Autoria própria (2021).

No total, 47,9\% (23) das internações no momento da admissão do paciente na pesquisa com suspeita de sepse na pesquisa ocorreram na UTI - Adulto, 27,1\% (13) na Clínica Amarela e 25\% (12) na Clínica Vermelha.

Tabela 2. Clínica de internação.

\begin{tabular}{cccc}
\hline & FREQUÊNCIA & PORCENTAGEM \\
\hline Amarela & 13 & $27.1 \%$ \\
Vermelha & 12 & $25.0 \%$ \\
UTI & 23 & $47.9 \%$ \\
\hline
\end{tabular}

Fonte: Autoria própria (2021).

Das comorbidades mais prevalentes $(\mathrm{n}=130), 23,86 \%$ (31) hipertensão; $21,54 \%$ (28) diabetes; 14,61\% (19) apresentavam cardiopatia; e, 10,77\% (14) disfunção renal, entre outras.

Tabela 3. Principais comorbidades apresentadas pelos sujeitos da pesquisa $(n=130)$.

\begin{tabular}{lcc}
\hline & PACIENTES & PORCENTAGEM \\
\hline Hipertensão arterial & 31 & $23,86 \%$ \\
Diabetes Mellitus & 28 & $21,54 \%$ \\
Insuficiência Cardíaca/ Cardiopatia & 19 & $14,61 \%$ \\
Disfunção Renal & 14 & $10,77 \%$ \\
Problemas pulmonares (DPOC, ex-tabagista, asma) & 7 & $5,38 \%$ \\
Doenças psiquiátricas (Alzheimer, Síndrome demencial, Transtorno de humor) & 7 & $5,38 \%$ \\
AVE & 5 & $3,84 \%$ \\
Neoplasia & 3 & $2,31 \%$ \\
Disfunção hepática & 3 & $2,31 \%$ \\
Ausência de comorbidade & 1 & $0,77 \%$ \\
Outros: Síndrome de imobilidade, hipotireoidismo, dislipidemia, úlcera gástrica, & \\
convulsão, febre reumática, ex-etilista, deficiência visual, hemiplegia, anemia, gastrite, & & $9,23 \%$ \\
encefalopatia & 12 & 2 \\
\hline
\end{tabular}

Fonte: Autoria própria (2021). 
Os Problemas respiratórios como pneumonia (nosocomial ou comunitária), Doença Pulmonar Obstrutiva Crônica (DPOC), insuficiência respiratória e asma foram apontadas como as complicações mais prevalentes como motivo inicial de internação hospitalar, sendo 29,2\% (14) das internações, seguidos de Sepse e Rebaixamento do Nível de Consciência, com 12,5\% (6) registros cada. Cetoacidose diabética; Acidente Vascular Encefálico; desconforto abdominal ou abdome agudo obstrutivo; Insuficiência renal ou Infecção do trato urinário; Lesões ou abscessos cutâneos; apresentaram 6,3\% (3) casos relatados cada.

Tabela 4. Relação do motivo da internação hospitalar.

\begin{tabular}{lcc}
\hline & FREQUÊNCIA & PORCENTAGEM \\
\hline Problemas Respiratórios & 14 & $29.2 \%$ \\
Sepse/septicemias & 6 & $12.5 \%$ \\
RNC - Rebaixamento do Nível de Consciência & 6 & $12.5 \%$ \\
Cetoacidose diabética & 3 & $6.3 \%$ \\
AVE - Acidente Vascular Encefálico & 3 & $6.3 \%$ \\
Desconforto abdominal, Abdome agudo obstrutivo, diarreia & 3 & $6.3 \%$ \\
Insuficiência renal ou Infecção do trato urinário & 3 & $6.3 \%$ \\
Lesão cutânea, abscesso cutâneo, celulite, furúnculo & 3 & $6.3 \%$ \\
Insuficiência ou doença hepática & 2 & $4.2 \%$ \\
Desconforto torácico & 2 & $4.2 \%$ \\
Transtorno mental e comportamental & 1 & $2.1 \%$ \\
Artrite piogênica, ostomielite & 1 & $2.1 \%$ \\
Hemoptise & 1 & $2.1 \%$ \\
\hline
\end{tabular}

Fonte: Autoria própria (2021).

Mais da metade dos casos suspeitos de sepse, 52,1\% (25) foi secundária a infecções pulmonares e trato respiratório, seguidos de 22,9\% (11) relacionados à foco urinário, 16,7\% (8) de foco abdominal, e 8,4\% (4) relacionados a outros focos (sendo este sangue/bacteremia, pele ou partes moles, articular ou associado a um cateter venoso central.

Tabela 5. Suspeita clínica do foco da infecção/sepse.

\begin{tabular}{lcc}
\hline & FREQUÊNCIA & PORCENTAGEM \\
\hline Pulmonar/respiratório & 25 & $52.1 \%$ \\
Urinário & 11 & $22.9 \%$ \\
Abdominal & 8 & $16.7 \%$ \\
Sangue/bacteremia ou associado ao Cateter Venoso Central & 2 & $4.2 \%$ \\
Pele/partes moles & 1 & $2.1 \%$ \\
Articular & 1 & $2.1 \%$ \\
\hline
\end{tabular}

Fonte: Autoria própria (2021).

Todos os diagnósticos foram feitos de forma empírica, de acordo com os achados clínicos do paciente que indicavam sepse.

Tabela 6. Tipo de diagnóstico.

FREQUENCIA PORCENTAGEM 
No momento da admissão na coleta, foi registrado qual suporte ou dispositivo o paciente estava utilizando $(\mathrm{n}=133)$. Desses, $22,6 \%$ (30) fez uso de sonda vesical de alívio urinário, o que aumenta o risco de infecções do trato urinário; 17,3\% (23) fez uso de alguma droga vasoativa; 15\% (20) fez uso de Cateter Venoso Central; 15\% (20) encontrava-se em sedação; 12,8\% (17) estava em processo de intubação orotraqueal em ventilação mecânica; 6,8\% (9) estava com traqueostomia e em ventilação mecânica acoplado a esse dispositivo; 4,5\% (6) em modo de ventilação espontânea para retirada intubação; 3,8\% (5) estava realizando diálise; e, 2,3\% (3) não utilizava nenhum suporte.

Tabela 7. Suporte admissional ( $\mathrm{n}=133)$.

\begin{tabular}{lcc}
\hline & PACIENTE & PORCENTAGEM \\
\hline Sonda Vesical de Demora & 30 & $22,6 \%$ \\
Droga vasoativa & 23 & $17,3 \%$ \\
Cateter Venoso Central & 20 & $15,0 \%$ \\
Sedação & 20 & $15,0 \%$ \\
Intubação orotraqueal + Ventilação Mecânica & 17 & $12,8 \%$ \\
Traqueostomia + Ventilação Mecânica & 9 & $6,8 \%$ \\
Intubação orotraqueal em modo espontâneo & 6 & $4,5 \%$ \\
Diálise & 5 & $3,8 \%$ \\
Ausência de suporte & 3 & $2,3 \%$ \\
\hline
\end{tabular}

Fonte: Autoria própria (2021).

Foi realizado o acompanhamento do tratamento farmacológico (sedação, drogas vasoativas e antibioticoterapia). Na maioria dos pacientes não houve necessidade de sedação $52,1 \%$ (25) e não fizeram uso de nenhuma de droga vasoativa $52,1 \%$ (25), durante o período da coleta de informações. Das substâncias mais utilizadas, o uso de noradrenalina foi a droga vasoativa mais prescrita, representando 41,7\% (20). A associação de fentanil com midazolam 27,1\% (13) foi o esquema mais utilizado para sedação.

\begin{tabular}{ccc}
\hline \multicolumn{3}{c}{ Tabela 8. Tratamento farmacológico adjuvante. } \\
\hline & DROGA VASOATIVA & \\
\hline & FREQUÊNCIA & PORCENTAGEM \\
\hline Não fez uso & 25 & $52.1 \%$ \\
Noradrenalina & 20 & $41.7 \%$ \\
Dobutamina & 1 & $2.1 \%$ \\
Noradrenalina/Dobutamina & 1 & $2.1 \%$ \\
Noradrenalina/Dobutamina/outra & 1 & $2.1 \%$ \\
\hline & FEDAÇÃO & \\
\hline Não fez uso & PREQUÊNCIA & PORCENTAGEM \\
\hline Fentanil + Midazolam & 27 & $56.3 \%$ \\
Midazolam & 13 & $27.1 \%$ \\
Dexmedetomidina & 3 & $6.3 \%$ \\
Midazolam/Fentanil/ Dexmedetomidina & 2 & $2.2 \%$ \\
Midazolan/ Fentanil & 1 & $2.1 \%$ \\
Fentanil/ Etomiado & 1 & $2.1 \%$ \\
\hline
\end{tabular}

Fonte: Autoria própria (2021). 
Para o tratamento da sepse, foi realizado o levantamento dos antibióticos prescritos de todos os pacientes e acompanhado o tempo de uso, além disso, calculou-se a média de dia de tratamento de cada antibiótico. Dentre eles, 21,3\% (26) o carbapenêmico Meropenem foi o antibiótico de maior escolha; em seguida, 13,9\% (17) a penicilina Piperacilina + Tazobactam; 13,1\% (16) a cefalosporina de $3^{a}$ geração Ceftriaxona; 8,2\% (10) o glicopeptídeo Vancomicina; 5,7 (7) o macrolídeo Azitromicina; 5,7\% (7) o imidazólico Metronidazol; 4,9\% (6) a penicilina Ampicilina + Sulbactam; 4,9\% (6) a Polimixina B; 4,1\% (5) a cefalosporina de $4^{\mathrm{a}}$ geração Cefepime; 4,1\% (5) a lincosamida Clindamicina; 3,3\% (4) o medicamento da classe dos triazóis Fluconazol; 2,5\% (3) o aminoglicosídeo Amicacina; e, 0,8\% (1) a quinolona Ciprofloxacino.

Tabela 9. Tratamento antimicrobiano.

\begin{tabular}{lcrrr}
\hline & $\begin{array}{c}\text { (N) PRESCRIÇÃO DE } \\
\text { ANTIBIÓTICOS }\end{array}$ & PORCENTAGEM & \multicolumn{2}{c}{ MÉDIAS DE DE USO } \\
DIAS \\
\hline Meropenem & 26 & $21,3 \%$ & 278 & 10,7 \\
Piperacilina + Tazobactam & 17 & $13,9 \%$ & 115 & 6,8 \\
Ceftriaxona & 16 & $13,1 \%$ & 77 & 4,8 \\
Vancomicina & 10 & $8,2 \%$ & 99 & 9,9 \\
Teicoplanina & 9 & $7,4 \%$ & 99 & 11,0 \\
Azitromicina & 7 & $5,7 \%$ & 29 & 4,1 \\
Metronidazol & 7 & $5,7 \%$ & 33 & 4,7 \\
Ampicilina + Sulbactam & 6 & $4,9 \%$ & 31 & 5,2 \\
Polimixina B & 6 & $4,9 \%$ & 60 & 10,0 \\
Cefepime & 5 & $4,1 \%$ & 28 & 5,6 \\
Clindamicina & 5 & $4,1 \%$ & 18 & 3,6 \\
Fluconazol & 4 & $3,3 \%$ & 44 & 11,0 \\
Amicacina & 3 & $2,5 \%$ & 12 & 4,0 \\
Ciprofloxacino & 1 & $0,8 \%$ & 11 & 11,0 \\
\hline
\end{tabular}

Fonte: Autoria própria (2021).

$\mathrm{Na}$ admissão do paciente na pesquisa, foi aplicado o SAPS3, Escore Simplificado da Fisiologia Aguda, que faz a análise de previsão de mortalidade, composto de 20 variáveis, desenvolvido em coorte mundial, onde o menor valor atribuído corresponde a 16 pontos e o maior é 217. O menor valor do índice SAPS 3 apresentando na pesquisa foi de 16 e o maior 74. A média do escore SAPS3 que discriminou sobreviventes e não sobreviventes foi 34,87. Já a média do SAPS3 para pacientes que evoluíram a óbito foi de 39,68 e a média de pacientes que tiveram alta foi de 31,91.

Além desse, cada acompanhamento do paciente foi aplicado a escala de coma Glasgow, que vai de 3 a 15 e registra o nível de consciência de um paciente e o escore de gravidade para avaliação sequencial de falha de órgão (Sequential Organ Failure Assessment - SOFA) que vai de 0 a 24, que acompanha a oxigenação sanguínea, contagem de plaquetas, hipotensão, bilirrubina, creatinina e a escala de coma de Glasgow.

Foi realizado o teste de comparação de médias Scott Knott a 5\% de probabilidade. As letras minúsculas que acompanham as médias indicam quais os melhores resultados considerando os dias avaliados (Tabela 10). 
Tabela 10. Escalas de acompanhamento.

\begin{tabular}{ccc|cc|c}
\hline $\begin{array}{c}\text { Acompanhamento do } \\
\text { paciente (dia) }\end{array}$ & $\begin{array}{c}\text { Glasgow } \\
\mathbf{3}-\mathbf{1 5} \\
\text { (média) }\end{array}$ & $\begin{array}{c}\text { Teste de } \\
\text { comparação de } \\
\text { médias Scott } \\
\text { Knott }\end{array}$ & $\begin{array}{c}\text { SOFA } \\
\mathbf{0}-\mathbf{2 4} \\
\text { (média) }\end{array}$ & $\begin{array}{c}\text { Teste de } \\
\text { comparação de } \\
\text { médias Scott Knott }\end{array}$ & $\begin{array}{c}\text { SAPS 3 } \\
\mathbf{1 6}-\mathbf{2 1 7} \\
\text { (média) }\end{array}$ \\
\hline D0 & 8,125 & $\mathrm{a}$ & 5,781667 & $\mathrm{a}$ & 34,708 \\
D3 & 7,3123 & $\mathrm{a}$ & 4,708333 & $\mathrm{~b}$ & \\
D5 & 6,625 & $\mathrm{a}$ & 3,729167 & $\mathrm{~b}$ & \\
D7 & 6,375 & $\mathrm{a}$ & 3,208333 & $\mathrm{~b}$ & \\
D10 & 6,291667 & $\mathrm{a}$ & 3,166667 & $\mathrm{~b}$ & \\
D14 & 3,5625 & $\mathrm{~b}$ & 1,729167 & & \\
\hline
\end{tabular}

*Teste Scott Knott a 5\%. Fonte: Autoria própria (2021).

Entre os pacientes com sepse, 50\% (24) paciente tiveram alta hospitalar e 45,8\% (22) foram a óbito.

Tabela 11. Óbito.

\begin{tabular}{lcc}
\hline & FREQUÊNCIA & PORCENTAGEM \\
\hline Não (alta) & 24 & $50.0 \%$ \\
Sim & 22 & $45.8 \%$ \\
Transferido de unidade hospitalar & 2 & $4.2 \%$ \\
\hline
\end{tabular}

Fonte: Autoria própria (2021).

Considerando o número de pacientes, foi possível aplicar a Correlação de Pearson (Emerson, 2015). Ao se comparar o valor do SOFA com a SAPS3, apresentou correlação moderada positiva. Quanto maior o valor de SOFA, maior foi o apresentado no escore SAPS3.

Já a correlação SAPS3 com óbito, representa uma correlação negativa (-1 a 1), com significância p menor que 0,05 , sendo a mortalidade predita pelo SAPS3 pouco proporcional ao apresentado pela mortalidade observada (taxa de óbito), em uma análise geral, representa baixa concordância e correlação já que faz uma análise de previsão de mortalidade. Esse viés de pesquisa pode ser justificado pela coleta de dados insuficientes para a realização dos cálculos das escalas de acompanhamento. Nem sempre estavam completamente disponíveis os resultados de exames ou a ausência de solicitação pela equipe médica dos mesmos.

Tabela 12. Correlação de Pearson entre SOFA X SAPS3 X Óbito.

\begin{tabular}{llrr}
\hline & & SOFA & ÓBITO \\
\hline \multirow{2}{*}{ SAPS 3} & Pearson Correlation & $0,534^{* *}$ & $-0,376^{* *}$ \\
& Sig. (2-tailed) & 0.00 & 0.01 \\
& Pearson Correlation & & -0.22 \\
& Sig. (2-tailed) & 0.13 \\
\hline
\end{tabular}

*Correlação de Pearson; **Correlation is significant at the 0.01 level (2-tailed). Fonte: Autoria própria (2021).

\section{Discussão}

A sepse é um problema de saúde pública capaz de aumentar as taxas de mortalidade e o tempo de permanência hospitalar e pode estar associada a características como idade, comorbidades e uso de dispositivos invasivos (Rhee, 2017 \& Sakr, 2018). Os resultados deste estudo trouxeram as principais características clínico-epidemiológicas e os desfechos relacionados à sepse em um hospital universitário no período de setembro de 2019 a fevereiro de 2020. 
Foi possível observar que a maioria dos pacientes eram idosos, do sexo feminino como também observado em outros estudos (Melo et al, 2019 \& Costa et al, 2019). O tempo de permanência hospitalar causada pela sepse gera forte impacto na evolução clínica do paciente e representa maiores custos para os serviços de saúde (Melo et al, 2019).

Foi constatada uma alta frequência de comorbidades entre os pacientes, em virtude da própria fisiologia do envelhecimento, com prevalência para hipertensão arterial, diabetes, cardiopatia e disfunção renal, ou seja, necessitavam de ajuste de dose de antimicrobianos. Em comparativo com a presente pesquisa, observou-se também em outros estudos com a mesma análise, apontaram que a maioria também eram portadores de hipertensão arterial sistêmica (Santos et al, 2016 \& Zonta et al, 2017 \& Melo et al, 2019).

Ao identificar a suspeita clínica de sepse, foram reconhecidos os focos das infecções. Mais da metade dos casos suspeitos de sepse, $52,1 \%$ (25) foi secundária a infecções pulmonares e trato respiratório, como também foi observado nos trabalhos de Cruz et al. (2016), Barros et al. (2016), Machado et al. (2017), Zonta et al. (2018), Morello et al. (2019) e Melo et al. (2019). Porém, é subjetivo afirmar que esse diagnóstico estava associado ao uso de ventilação mecânica pois não havia informações suficientes em prontuário para chegar a tal conclusão. $\mathrm{O}$ mesmo pode-se dizer sobre a segunda infecção mais prevalente, que estava relacionada à infecção urinária, não é possível generalizar que os resultados estão associados ao uso de dispositivos invasivos (cateter vesical de demora).

Devido ao aumento da morbimortalidade, recomenda-se realizar hemocultura de todos os pacientes com suspeita de sepse ou choque séptico, independente do foco infeccioso, antes da administração da terapia antimicrobiana empírica. Para os pacientes que já estão submetidos à antibioticoterapia, devem ser realizadas culturas, desde que sejam ponderadas as limitações, devido a probabilidade de resultados falso-negativos, pelo uso prévio de antibióticos (Ilas, 2018).

A dificuldade em se realizar uma confirmação imediata em relação ao diagnóstico microbiológico através de antibiograma, como mostrado na tabela 6 , pode estar relacionada a demora da solicitação e coleta de cultura ou a demora do resultado, sem resultados prévios parciais, já que o exame é feito de forma terceirizada, sendo as amostras encaminhadas para laboratório externo. Desse modo, o diagnóstico e o tratamento são realizados tardiamente, colaborando para a cronicidade da sepse, para o aumento do tempo de internação e elevação dos índices de mortalidade (Zonta et al, 2017).

Atraso no reconhecimento da sepse e transferência tardia para as UTI são ocorrências comuns no Brasil. Outros estudos demonstraram que a adesão a um regime antibiótico foi associados à sobrevivência, reforçando evidências anteriores de que sugere que este é um passo fundamental no tratamento da sepse (Machado, 2017).

Neste estudo, os $\beta$-lactâmicos foram os mais utilizados com destaque para os carbapenêmicos, como mostrado na tabela 9. Este número pode ser explicado pela necessidade de cobertura de amplo espectro, uma vez que a predominância foi de pacientes com identificação do foco infeccioso associado a prevalência de queixas respiratórias.

Protocolos recomendam individualizar o esquema terapêutico considerando as alterações farmacodinâmicas dos antibióticos no indivíduo crítico, onde é essencial o ajuste estrito do antimicrobiano de acordo com o sítio e o perfil microbiano. A utilização de medidas de função renal pode ser um dos critérios para adequação de drogas que apresentam maior probabilidade de sobrecarga renal (Ilas, 2018).

O uso racional dos antimicrobianos é imprescindível porque o atraso em seu início pode aumentar a mortalidade, mas a exposição desnecessária é potencialmente prejudicial. Por isso deve ser levado em conta o tempo mínimo necessário para eliminar a infecção e assim, limitar o potencial risco de resistência bacteriana (Russel, 2018).

De acordo com o estudo observacional sobre a epidemiologia da sepse em unidades de terapia intensiva brasileiras de Machado et. al. (2017), os resultados mostram que a sepse representa um enorme fardo nas configurações de recursos limitados. Políticas nacionais que visam reduzir esse ônus são urgentemente necessárias e devem basear-se em medidas 
preventivas, educação continuada, incluindo assistência médica associada prevenção de infecções e iniciativas de melhoria da qualidade, visando melhorar o reconhecimento precoce e tratamento de sepse.

$\mathrm{Na}$ maioria dos estudos, a sepse vem acometendo um grande número de pacientes, mas em contrapartida, essa patologia apesar de amplamente descrita na literatura é pouco discutida na prática clínica, e, muitas vezes, a causa de morte é atribuída à patologia de base e não à sepse (Zonta, 2018).

$\mathrm{O}$ viés apresentado na correlação da taxa de óbito com as escalas aplicadas nesta pesquisa pode ser justificado, pois nem sempre havia todas as informações de exames disponíveis para o cálculo dos escores de acompanhamento de disfunção orgânica ou pela ausência de solicitação da equipe médica dos mesmos.

O ILAS possui o maior número de dados de sepse registrados em hospitais brasileiros, embora conte apenas com cooperação voluntária de alguns locais para coleta de dados. No Brasil, dentro do mesmo hospital, de acordo com o local (enfermaria, pronto-socorro ou UTI), a mortalidade varia de $29 \%$ a 64\%. Essa discrepância também é observada quando se comparam hospitais privados e públicos: $34,5 \%$ dos pacientes com sepse morrem em hospitais privados, enquanto nos hospitais públicos a taxa média de mortalidade é de 55,7\% (Morello, 2018).

\section{Considerações Finais}

Pacientes idosos e pacientes com períodos prolongados de internação são mais vulneráveis e acometidos pela sepse. É possível observar que o foco de infecção mais encontrado foi pulmonar e urinário, que podem estar associados a problemas prévios ou uso de dispositivos invasivos, como ventilação mecânica e sonda vesical de demora, respectivamente. A dificuldade em se realizar uma confirmação imediata em relação ao diagnóstico microbiológico através de antibiograma, pode estar relacionada a demora da solicitação e coleta de cultura ou a demora do resultado sem resultados prévios parciais, o que reforçam a importância da implementação de práticas mais efetivas e eficientes.

Por ser um estudo prospectivo, facilitou acompanhar os tratamentos utilizados, o tempo de uso e possíveis substituições. Entre os tratamentos antimicrobianos mais utilizados estão o Meropenem, a Piperacilina + Tazobactam e Ceftriaxona. Devido à dificuldade de diagnóstico, a escolha de antibióticos de amplo espectro foi mais identificada para cobrir os possíveis e diversos tipos de patógenos suspeitos, o que indica que estimular a interação multiprofissional na instituição para identificar precocemente a sepse e ampliar os estudos em relação a tratamento empírico racional pode reduzir os efeitos da resistência bacteriana. Em contraste com as escalas de indicadores de gravidade, a taxa de mortalidade identificada é preocupante e reforça a necessidade de identificar os fatores associados a essas disparidades, que podem ser consequência de atraso na hospitalização ou no diagnóstico, conduta terapêutica inadequada, entre outros. Essas análises podem ser usadas para formular políticas locais para o gerenciamento desse grave problema de saúde pública.

Recomenda-se administrar terapia antimicrobiana adequada e precoce, assim que for feito diagnóstico de sepse ou choque séptico. Terapia empírica de amplo espectro deve ser utilizada nos pacientes com sepse ou choque séptico, com o objetivo de oferecer melhor cobertura antimicrobiana precoce para o paciente (Ilas, 2018). Na escolha da terapia de amplo espectro deve-se considerar os seguintes critérios: o foco primário da infecção, a suscetibilidade dos patógenos conforme o local de aquisição da infecção (hospital ou comunidade), infecções prévias e uso recente de antimicrobianos.

Pode-se optar pelo uso de $\beta$-lactâmicos de amplo espectro em monoterapia, que apresentam superioridade em relação ao uso de um espectro mais restrito de $\beta$-lactâmicos combinados aos aminoglicosídeos (Ilas, 2018).

Entre os pacientes com sepse, $45,8 \%$ (22) foram a óbito, valor observado próximo ou superior a outros estudos (Costa, 2019 \& Morello, 2019). A mortalidade observada neste estudo é preocupante, por isso a importância de implantar um protocolo de sepse nas instituições (Pedrosa, 2018 \& De Menezes, 2019). O resultado apresentado por este estudo, encontra-se 
próximo ao identificado pelo trabalho de Morello (2019), que realizou um estudo parecido em uma instituição de ensino com atendimento terciário público.

\section{Referências}

Azevedo, L. C. P \& Machado, F. R. M. (2019). Sepse. (2a ed.). Atheneu: 3-13.

Barros, L. L. S., Maia, C. S. F \& Monteiro, M. C. (2016). Fatores de risco associados ao agravamento de sepse em pacientes em Unidade de Terapia Intensiva. Cad. Saúde Colet, 24(4), 388-396.

Bouadma, L. et al. (2010). Use of procalcitonin to reduce patients' exposure to antibiotics in intensive care units (PRORATA trial): a multicentre randomised controlled trial. The Lancet, 375(9713), 463-474.

Costa, M. B. V., de Azevedo Ponte, K. M., da Frota, K. C., \& Moreira, A. C. A. (2019). Características epidemiológicas de pacientes com sepse em unidade de terapia intensiva. Revista de Epidemiologia e Controle de Infecção, 9(4), 310-315.

Cruz, L. L \& Macedo, C. C. (2016). Perfil epidemiológico da sepse em hospital de referência no interior do Ceará. Id On Line Revista Multidisciplinar e de Psicologia, 10(29), 71-99.

De Menezes, L. E. F. J. et al. (2019). Perfil epidemiológico e análise da efetividade para prevenção de óbitos de pacientes inseridos em protocolo de sepse. Revista da Sociedade Brasileira de Clínica Médica, 17(1), 25-30.

Do Nascimento Borges, A. C. et al. (2020). Epidemiology and pathophysiology of sepsis: an review. Research, Society And Development, 9(2), 187922112.

Emerson, R. W. (2015). Causation and Pearson's correlation coefficient. Journal Of Visual Impairment \& Blindness, 109(3), $242-244$.

Ilas, Instituto Latino Americano De Sepse. (2018). Implementação De Protocolo Gerenciado De Sepse - Protocolo Clínico. Atendimento ao paciente adulto com sepse/choque séptico. <https://www.ilas.org.br/assets/arquivos/ferramentas/protocolo-de-tratamento.pdf >.

Machado, F. R. et al. (2017). The epidemiology of sepsis in Brazilian intensive care units (the Sepsis PREvalence Assessment Database, SPREAD): an observational study. The Lancet Infectious Diseases, 17(11), 1180-1189.

Machado, F. R. et al. (2016). Chegando a um consenso: vantagens e desvantagens do Sepsis 3 considerando países de recursos limitados. Rev. bras. ter. intensiva, 28(4), 361-365, Dec.

Melo, M. S. et al. (2019). Aspectos clínicos e epidemiológicos de pacientes internados com sepse em um hospital privado. Revista Enfermagem Atual InDerme, 90(28).

Pedrosa K. K. A., Oliveira S.A \& Machado R.C. (2018). Validation of a care protocol for the septic patient in the Intensive Care Unit. Rev Bras Enferm; 71(3):1106-14

PDCSU. Primeiras Diretrizes Clínicas Na Saúde Suplementar. (2010). Versão preliminar / organizado por Agência Nacional de Saúde Suplementar, Associação Médica Brasileira. - Rio de Janeiro: ANS, 2010.

Rhee C., Dantes R., Epstein L., Murphy J., Seymour C \& Iwashyna T. (2017). Incidence and Trends of Sepsis in USHospitals Using Clinical vs Claims Data, 2009-2014. JAMA.

Russel J. A., Rush B \& Boyd J. (2018). Pathophysiology of Septic Schock. Crit Care Med.

Sakr Y., Jaschinski U., Wittebole X., Szakmany T., Lipman J \& Silva San. (2018). Sepsis in Intensive Care Unit Patients: Worldwide Data From the Intensive Care over Nations Audit. Open Forum Infect Dis.

Singer, M. et al. (2016). The Third International Consensus Definitions for Sepsis and Septic Shock (Sepsis-3). JAMA;315(8):801-10.

Santos, Am., Souza, G. R. B \& Oliveira, A. M. L. (2016). Sepse em adultos na unidade de terapia intensiva: características clínicas. Arq Med Hosp Fac Cienc Med Santa Casa São Paulo. Surviving Sepsis Campaign: International Guidelines for Management of Sepsis and Septic Shock: Volume 45, N 3.

Zonta, F. N. S. et al. (2018). Epidemiological and clinical characteristics of sepse in a public hospital of Paraná. 